Proceedings of the 2005 Winter Simulation Conference

M. E. Kuhl, N. M. Steiger, F. B. Armstrong, and J. A. Joines, eds.

\title{
PROCEDURES FOR FEASIBILITY DETECTION IN THE PRESENCE OF MULTIPLE CONSTRAINTS
}

\author{
Demet Batur \\ Seong-Hee Kim \\ School of Industrial and Systems Engineering \\ Georgia Institute of Technology \\ Atlanta, GA 30332-0205, U.S.A.
}

\begin{abstract}
In this paper, we address the problem of finding a set of feasible or near-feasible systems among a finite number of simulated systems in the presence of stochastic constraints. Andradóttir, Goldsman, and Kim (2005) present a procedure that detects feasibility of systems in the presence of one constraint with a pre-specified probability of correctness. We extend their procedure to the case of multiple constraints by the use of the Bonferroni inequality. Unfortunately, the resulting procedure tends to be very conservative when the number of systems or constraints is large. As a remedy, we present a screening procedure that uses an aggregated observation, which is a linear combination of the collected observations across stochastic constraints. Then, we present an accelerated procedure that combine the extension of Andradóttir, Goldsman, and Kim (2005) with the procedure that uses aggregated observations. Some experimental results that compare the performance of the proposed procedures are presented.
\end{abstract}

\section{INTRODUCTION}

Ranking and selection (R\&S) performs comparisons among a finite number of simulated systems. It mainly focuses on finding the best or near-best systems with the largest or smallest expected primary performance measure by either maximizing or minimizing an objective function. However, very little work has been done for solving an optimization problem in the presence of stochastic constraints on some secondary performance measures. Butler, Morrice and Mullarkey (2001) handle multiple performance measures and Santner and Tamhane (1984) propose a two-stage procedure with a constraint on variance. However, their methods are either very hard to apply in practice or focused on only a special case. Andradóttir, Goldsman, and Kim (2005) present a R\&S procedure that determines the feasibility of a system for one stochastic constraint. This procedure can handle a general stochastic constraint on a secondary performance measure and determines the feasibility of systems in consideration correctly with high probability. Yet, the work needs to be further extended to multiple constraints.

In this paper, we tackle the problem of determining a set of feasible or near-feasible systems that satisfy a number of stochastic constraints, especially, when the number of systems or constraints is large. Determining the best feasible system, which minimizes or maximizes the primary performance measure, from the resulting set is beyond the scope of this paper.

Any R\&S procedure that checks feasibility in the presence of one stochastic constraint can easily be extended to the case of multiple constraints by the use of the Bonferroni inequality. We call the extended procedure $\mathcal{F}_{B}$. In this paper, we only focus on the extended version of the feasibility check procedure by Andradóttir, Goldsman, and Kim (2005). Unfortunately, the conservativeness of the Bonferroni inequality is well-known and this conservativeness becomes more serious when the number of systems or constraints is large. To lessen it, we present a screening procedure that accelerates elimination of infeasible systems. The idea is to use an aggregated observation, which is a linear combination of observations across the stochastic constraints in consideration. How does this idea help the elimination of the infeasible systems?: When there are multiple constraints, a system is feasible if all the constraints are satisfied. If at least one constraint is violated, then the system is infeasible and eliminated immediately regardless of the feasibility of the other constraints. When infeasibility occurs in one or two constraints, then it might be more effective to keep a feasibility check test for each constraint. However, if a number of constraints contribute to infeasibility together, then an aggregated observation of the collected observations corresponding to each constraint would make it easier to detect infeasibility. From this motivation, we design a screening procedure that uses an aggregated observation for feasibility check and show how to combine this idea with $\mathcal{F}_{B}$ to accelerate the efficiency of $\mathcal{F}_{B}$. 


\section{Batur and Kim}

The paper is organized as follows: In Section 2, the problem is formulated and notation and definition are given. Section 3 provides the $\mathcal{F}_{\mathcal{B}}$ procedure-an extended procedure of the feasibility check procedure due to Andradóttir, Goldsman, and Kim (2005) to multiple constraints using the Bonferroni inequality - and Section 4 presents an accelerated version of $\mathcal{F}_{B}$. Some experimental results on independent and identically distributed (i.i.d.) multivariate normal data are presented in Section 5, followed by a conclusion in Section 6.

\section{PROBLEM}

In this section, we define our problem and notation for the paper. Our problem is to determine a set of feasible or near-feasible systems from $k$ simulated systems that satisfy $s$ stochastic constraints. Let $Y_{i \ell j}$, for $i=1,2, \ldots, k$, $\ell=1,2, \ldots, s, j=1,2, \ldots$, denote an observation from the $j$ th replication associated with the $\ell$ th performance measure (or the $\ell$ th constraint) from the $i$ th system, and let $\mathbf{Y}_{i j}=\left(Y_{i 1 j}, Y_{i 2 j}, \ldots, Y_{i s j}\right)^{\prime}$ be the vector of $j$ th observation across all $s$ performance measures from system $i$. The expected performance measure of system $i$ is defined as $\mathbf{y}_{i}=\mathrm{E}\left[\mathbf{Y}_{i j}\right]=\left(y_{i 1}, y_{i 2}, \ldots, y_{i s}\right)^{\prime}$ where $\mathrm{E}\left[Y_{i \ell j}\right]=y_{i \ell}$ for $\ell=1,2, \ldots, s$ and $j=1,2, \ldots$ We assume

$$
\mathbf{Y}_{i j}=\left[\begin{array}{l}
Y_{i 1 j} \\
Y_{i 2 j} \\
\vdots \\
Y_{i s j}
\end{array}\right] \stackrel{\text { i.i.d. }}{\sim} \mathbf{M N}\left(\mathbf{y}_{i}, \Sigma_{i}\right)
$$

where $\sim$ implies 'are distributed as', MN implies multivariate normal, and $\Sigma_{i}$ is the variance-covariance matrix of $\mathbf{Y}_{i j}$. Further, we assume that $\mathbf{Y}_{i j}$ and $\mathbf{Y}_{v j}$ are independent for $i \neq v$ (i.e., no common random numbers). Throughout, we write vectors in boldface, random variables in upper case, and their realizations in lower case. If we take withinreplication averages as basic observations, then $Y_{i \ell j}$ are likely to be approximately normally distributed. Performance measures from a system are likely to be correlated in practice such as waiting times and the number of jobs waiting. Therefore, the assumption of multivariate normal random variables is plausible.

In order for a system to be feasible, the vector of mean performance measures has to be less than a constant vector $\mathbf{Q}=\left(Q_{1}, Q_{2}, \ldots, Q_{s}\right)^{\prime}$. More specifically, system $i$ will be feasible if $\mathbf{y}_{i} \leq \mathbf{Q}$. Unfortunately, for stochastic systems, it is impossible to guarantee identifying all feasible systems that satisfy $s$ stochastic constraints. Instead Andradóttir, Goldsman, and Kim (2005) introduce tolerance level which is similar to the indifference-zone (IZ) parameter and we adopt the same approach in this paper. For each constraint, a decision maker will be asked to give a range around $Q_{\ell}$, say, $\left(Q_{\ell, \text { low }}, Q_{\ell, \text { up }}\right)$ such that $Q_{\ell, \text { low }} \leq Q_{\ell} \leq Q_{\ell, \text { up }}$ and $Q_{\ell, \text { low }}<Q_{\ell, \text { up }}$. Let $\mathbf{Q}_{\text {low }}=\left(Q_{1, \text { low }}, \ldots, Q_{s, \text { low }}\right)^{\prime}$ and $\mathbf{Q}_{u p}=\left(Q_{1, u p}, \ldots, Q_{s, u p}\right)^{\prime}$. Then three regions are defined for the constraints we consider:

- $\quad \mathbf{y}_{i} \leq \mathbf{Q}_{\text {low }}$ : This is the desirable region. If system $i$ is in this range, then it is feasible.

- $\quad\left(\mathbf{y}_{i}<\mathbf{Q}_{\text {up }}\right) \backslash\left(\mathbf{y}_{i} \leq \mathbf{Q}_{\text {low }}\right)$ : This is the acceptable region. If system $i$ is in this range, it is either feasible or infeasible and can be declared feasible or infeasible regardless of its true feasibility.

- $\quad\left(y_{i 1} \geq Q_{1, u p}\right) \cup\left(y_{i 2} \geq Q_{2, u p}\right) \cup \ldots \cup\left(y_{i s} \geq Q_{s, u p}\right)$ : This is the unacceptable region. If system $i$ is in this range, then it is infeasible and should be eliminated.

Furthermore, we can define the following three sets for the constraints in consideration:

$S_{D}=$ the set of all desirable systems;

$S_{A}=$ the set of all acceptable systems;

$S_{U}=$ the set of all unacceptable systems.

For given $Q_{\ell, \text { low }}$ and $Q_{\ell, \text { up }}$, we define $q_{\ell}$ and $\epsilon_{\ell}$ as $q_{\ell}=\left(Q_{\ell, \text { low }}+Q_{\ell, \text { up }}\right) / 2$ and $\epsilon_{\ell}=\left(Q_{\ell, \text { up }}-Q_{\ell, \text { low }}\right) / 2$, respectively. In this setup, $Q_{\ell, \text { low }}=q_{\ell}-\epsilon_{\ell}$ and $Q_{\ell, u p}=$ $q_{\ell}+\epsilon_{\ell}$ and our procedures will be presented in terms of $q_{\ell}$ and $\epsilon_{\ell}$. The parameter $q_{\ell}$, called the target value of the $\ell$ th constraint, behaves as a cut-off point between feasible and infeasible systems for the $\ell$ th constraint. Parameter $\epsilon_{\ell}$ is the tolerance level of the $\ell$ th constraint, indicating how much we are willing to be off and above from $q_{\ell}$. Figure 1 shows the desirable $(D)$, acceptable $(A)$, and unacceptable $(U)$ regions when there are two stochastic constraints.

Further, we let $\mathbf{q}=\left(q_{1}, q_{2}, \ldots, q_{s}\right)^{\prime}$ and $\mathbf{E}=$ $\left(\epsilon_{1}, \epsilon_{2}, \ldots, \epsilon_{s}\right)^{\prime}$ be the vector of target values and tolerance levels for the $s$ constraints, respectively. A correct decision (CD) is defined as the event that a set $F$ returned by a procedure as a set of feasible systems is $S_{D} \subset F \subset\left(S_{D} \cup S_{A}\right)$.

\section{MULTIPLE FEASIBILITY CHECK PROCEDURE}

In this section, we consider Algorithm I presented in Andradóttir, Goldsman, and Kim (2005) and extend it to the case of multiple constraints. Algorithm I handles only one constraint and requires tolerance level $\epsilon$ and target value $q$ for the constraint. It is fully sequential with a triangular boundary, that is sometimes called a triangular continuation region. In a fully sequential procedure, one samples one basic observation from each survivor at each stage and compute the partial sum of the difference between the observation and the target value $q$ up to the current stage. If this partial sum stays within the boundary, sampling continues. Otherwise, the procedure stops and a decision is made 


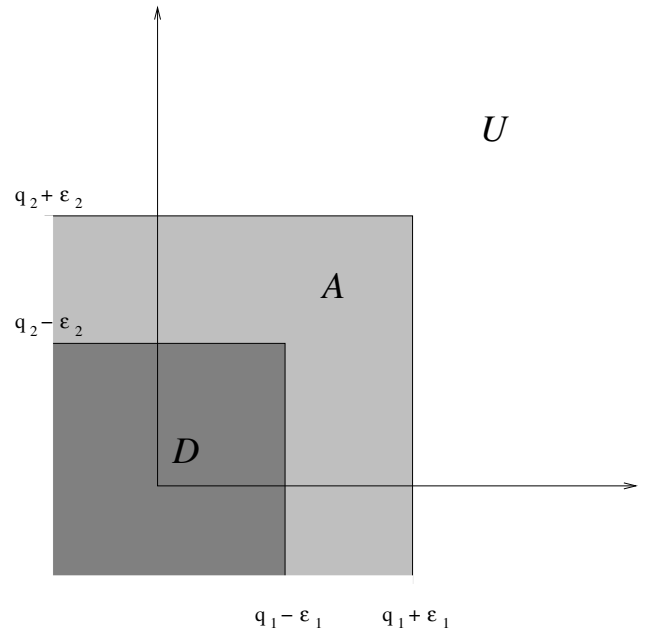

Figure 1: Desirable $(D)$, Acceptable $(A)$, and Unacceptable $(U)$ Regions in the Presence of Two Stochastic Constraints

depending on through which side of the boundary the exit occurs. For example, Figure 2 shows the triangular continuation region of Algorithm I. The horizontal and vertical axes in the figure denote stage number, $r$, and partial sums, $\sum_{j=1}^{r}\left(X_{i j}-q\right)$, respectively. If the exit occurs through the upper boundary, we conclude that $\mu_{i}>q$ and the system is infeasible. On the other hand, if the exit occurs through the lower boundary, we conclude that $\mu_{i} \leq q$ and the system is feasible. Algorithm I guarantees the event of CD with at least $1-\alpha$ probability. The $\mathcal{F}_{\mathcal{B}}$ procedure given below is the extension of Algorithm I to the case of $s$ constraints via the Bonferroni inequality.

$\sum_{j=1}^{r}\left(X_{i j}-q\right)$

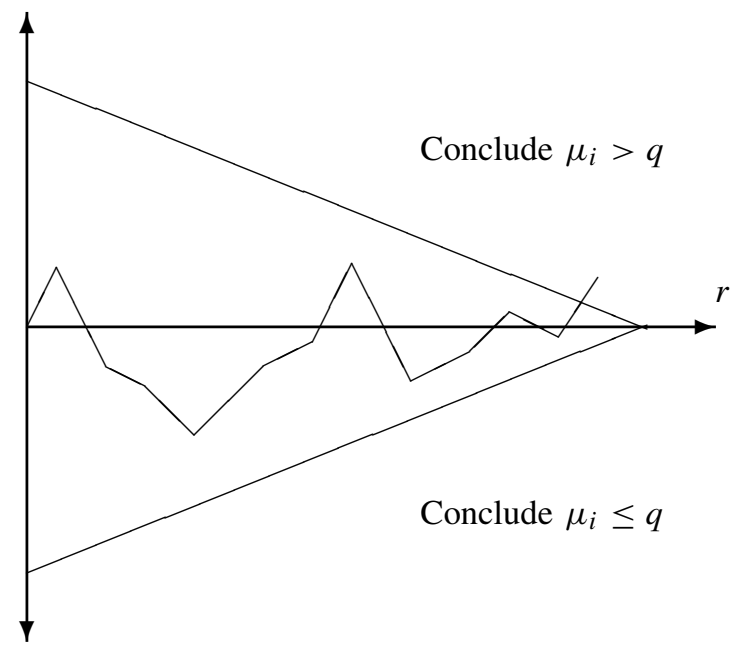

Figure 2: Continuation Region for the Constraint $\mu_{i} \leq q$ in Algorithm I of Andradóttir, Goldsman, and Kim (2005)

\section{Algorithm of $\mathcal{F}_{\mathcal{B}}$ :}

Setup: Choose confidence level $1-\alpha$, vector of tolerance levels $\mathbf{E}=\left(\epsilon_{1}, \epsilon_{2}, \ldots, \epsilon_{s}\right)^{\prime}$, and first stage sample size $n_{0} \geq 2$. Find $\eta$ as the solution to the equation

$g(\eta) \equiv \frac{1}{2}(1+2 \eta)^{-\left(n_{0}-1\right) / 2}=1-(1-\alpha)^{1 /(k s)}$.

Initialization: Let $I=\{1,2, \ldots, k\}, F=\emptyset$, and $S_{i}=\emptyset, i=1,2, \ldots, k$, be the set of undetermined systems, the set of systems declared 'feasible', and the set of constraint indexes of system $i$ that are already checked as feasible for system $i$, respectively. Let $h^{2}=2 \eta \times\left(n_{0}-1\right)$.

Obtain observations $\mathbf{Y}_{i j}, j=1,2, \ldots, n_{0}$, from each system $i$. For each system $i$ and constraint $\ell=1,2, \ldots, s$, compute the sample variance

$$
S_{i \ell}^{2}=\frac{1}{n_{0}-1} \sum_{j=1}^{n_{0}}\left(Y_{i \ell j}-\overline{Y_{i l}}\left(n_{0}\right)\right)^{2},
$$

where $\overline{Y_{i l}}\left(n_{0}\right)$ is the sample average of the first $n_{0}$ observations associated with constraint $\ell$ from system $i$.

Set the number of observations $r=n_{0}$ and go to Feasibility Check.

Feasibility Check: Let

$$
R(r ; a, b, c)=\max \left\{0, \frac{a}{2}\left(\frac{b c}{a^{2}}-r\right)\right\}
$$

for $a, b, c>0$.

For each $i \in I$ and any $\ell \notin S_{i}, \ell=1,2, \ldots, s$, if

$$
\sum_{j=1}^{r}\left(Y_{i \ell j}-q_{\ell}\right) \geq+R\left(r ; \epsilon_{\ell}, h^{2}, S_{i \ell}^{2}\right),
$$

then eliminate $i$ from $I$; else if

$$
\sum_{j=1}^{r}\left(Y_{i \ell j}-q_{\ell}\right) \leq-R\left(r ; \epsilon_{\ell}, h^{2}, S_{i \ell}^{2}\right),
$$

then add $\ell$ to $S_{i}$.

For each $i \in I$, if $\left|S_{i}\right|=s$, then move $i$ from $I$ and to $F$.

Stopping Rule: If $|I|=0$, then return $F$ as a set of feasible systems.

Otherwise, take one additional observation $\mathbf{Y}_{i, r+1}$ from each system $i \in I$. Then, set $r=r+1$ and go to Feasibility Check. 
The $\mathcal{F}_{\mathcal{B}}$ procedure eliminates all unacceptable systems and returns a set of all desirable systems, possibly with some acceptable systems, with probability at least $1-\alpha$. The full proof is given in Batur and Kim (2005).

\section{ACCELERATED FEASIBILITY CHECK PROCEDURE}

When the number of systems or constraints is large, the $\mathcal{F}_{\mathcal{B}}$ procedure becomes very conservative in terms of the number of observations required and the actual probability of $\mathrm{CD}$ (PCD) because of the Bonferroni inequality. To lessen this conservativeness, we design a screening procedure in which basic observations associated with each constraint are aggregated into one observation by a linear combination $\mathbf{A}^{\prime} \mathbf{Y}_{i j}$ where $\mathbf{A}=\left(a_{1}, a_{2}, \ldots, a_{s}\right)^{\prime}$ is a vector of positive constants. Since $\mathbf{Y}_{i j}, j=1,2, \ldots$, are assumed to be i.i.d. multivariate normally distributed, the aggregated observations $\mathbf{A}^{\prime} \mathbf{Y}_{i j}$ are also i.i.d. normally distributed. Therefore, we can apply $\mathcal{F}_{\mathcal{B}}$ directly to the aggregated observations with aggregated tolerance level $\epsilon^{a}=\mathbf{A}^{\prime} \mathbf{E}$ and aggregated target value $q^{a}=\mathbf{A}^{\prime} \mathbf{q}$. The difficulty is that aggregation with $\mathbf{A}^{\prime} \mathbf{Y}_{i j}, \epsilon^{a}$, and $q^{a}$ results in different desirable, acceptable, and unacceptable regions from those defined by the original individual constraints.

More specifically, let $D^{a}, A^{a}$, and $U^{a}$ denote desirable, acceptable, and unacceptable regions defined by aggregation with a vector A. Shaded triangles of Figure 3 show the three regions for the aggregated constraint while rectangles show those for the original individual constraints when there are two constraints. The vector $\mathbf{A}$ is usually chosen so that $U^{a}$ and $A^{a}$ do not contain any desirable system in order to avoid declaring a desirable system as infeasible. Batur and Kim (2005) discuss the choice of $\mathbf{A}$ in more detail. As one can see from Figure 3, all systems in $U^{a}$ fall into the unacceptable region $U$ in terms of the original constraints, so the screening procedure with aggregated observations is likely to eliminate systems in $U$ only. However, $A^{a}$ and $D^{a}$ contain some unacceptable systems in terms of the original constraints. Therefore, it is possible that a system declared as feasible by the screening procedure with aggregated observations is actually an unacceptable system in $U$. This implies that we can confidently eliminate a system if the system is declared as infeasible by the screening procedure with aggregated observations, but a decision that a system is feasible by the procedure is untrustworthy because $D^{a}$ and $A^{a}$ contain unacceptable systems in terms of the original constraints. Therefore, the screening procedure with aggregated observations can not be used solely to make the feasibility decision but can help accelerate elimination of unacceptable systems in the presence of multiple constraints, especially when a number of constraints contribute infeasibility of the system. To utilize the efficiency of the screening procedure with aggregated observations in elim-

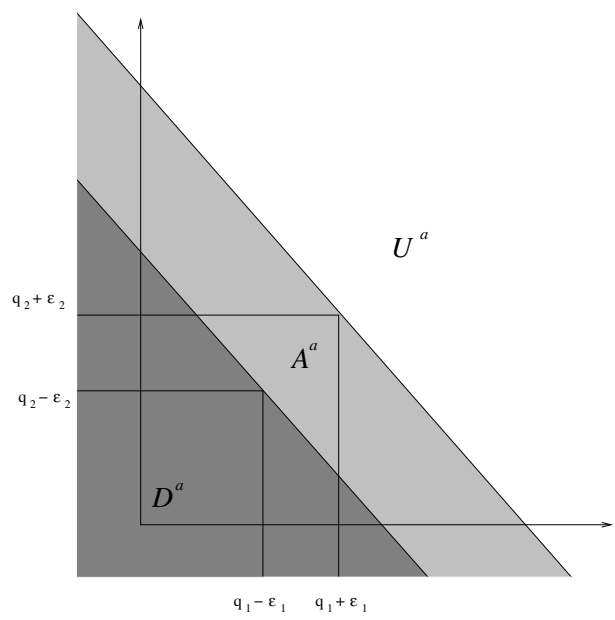

Figure 3: $D^{a}, A^{a}$, and $U^{a}$ Regions for the Aggregated Measure in the Presence of Two Stochastic Constraints

inating unacceptable systems, we combine it with the $\mathcal{F}_{\mathcal{B}}$ procedure. The combined accelerated procedure is called the $\mathcal{F}_{\mathcal{A}}$ procedure and the steps of the procedure are given below.

\section{Algorithm of $\mathcal{F}_{\mathcal{A}}$ :}

Setup: Choose confidence level $\alpha_{0}$ and $\alpha_{1}$, vector of tolerance levels $\mathbf{E}=\left(\epsilon_{1}, \epsilon_{2}, \ldots, \epsilon_{s}\right)^{\prime}$, and first stage sample size $n_{0} \geq 2$. Compute $\epsilon^{a}=\mathbf{A}^{\prime} \mathbf{E}$ and $q^{a}=\mathbf{A}^{\prime} \mathbf{q}$ where $\mathbf{A}=\left[A_{\ell}\right]_{\ell=1,2, \ldots, s}$ such that $A_{\ell}=\prod_{j=1, j \neq \ell}^{s} \epsilon_{j}$ (see Batur and Kim 2005 for more detail about the choice of $\mathbf{A}$ ). Find $\eta_{0}$ as the solution to the equation

$$
g\left(\eta_{0}\right)=1-\left(1-\alpha_{0}\right)^{1 / k}
$$

and $\eta_{1}$ as the solution to the equation

$$
g\left(\eta_{1}\right)=1-\left(1-\alpha_{1}\right)^{1 /(k s)}
$$

Initialization: $\quad$ Let $I=\{1,2, \ldots, k\}, F=\emptyset$, and $S_{i}=$ $\emptyset, i=1,2, \ldots, k$, be the set of undetermined systems, the set of systems declared 'feasible', and the set of constraint indexes of system $i$ that are already checked as feasible, respectively. Let $h_{0}^{2}=2 \eta_{0} \times\left(n_{0}-1\right)$ and $h_{1}^{2}=2 \eta_{1} \times\left(n_{0}-1\right)$.

Obtain observations $\mathbf{Y}_{i j}, j=1,2, \ldots, n_{0}$, from each system $i$. Compute $Y_{i j}^{a}=\mathbf{A}^{\prime} \mathbf{Y}_{i j}$. For each system $i$, compute the sample variance

$$
S_{i}^{2}=\frac{1}{n_{0}-1} \sum_{j=1}^{n_{0}}\left(Y_{i j}^{a}-\overline{Y_{i}^{a}}\left(n_{0}\right)\right)^{2},
$$




\section{Batur and Kim}

where $\overline{Y_{i}^{a}}\left(n_{0}\right)$ is the sample average of the first $n_{0}$ aggregated observations from system $i$.

For each system $i$ and constraint $\ell=1,2, \ldots, s$, compute the sample variance

$$
S_{i \ell}^{2}=\frac{1}{n_{0}-1} \sum_{j=1}^{n_{0}}\left(Y_{i \ell j}-\overline{Y_{i l}}\left(n_{0}\right)\right)^{2},
$$

where $\overline{Y_{i l}}\left(n_{0}\right)$ is the sample average of the first $n_{0}$ observations associated with constraint $\ell$ from system $i$.

Set the number of observations $r=n_{0}$ and go to Feasibility Check.

Feasibility Check: $\quad$ For each system $i \in I$, if

$$
\sum_{j=1}^{r}\left(Y_{i j}^{a}-q^{a}\right) \geq+R\left(r ; \epsilon^{a}, h_{0}^{2}, S_{i}^{2}\right)
$$

then eliminate $i$ from $I$. Otherwise for each $i \in I$ and any $\ell \notin S_{i}, \ell=1,2, \ldots, s$, if

$$
\sum_{j=1}^{r}\left(Y_{i \ell j}-q_{\ell}\right) \geq+R\left(r ; \epsilon_{\ell}, h_{1}^{2}, S_{i \ell}^{2}\right),
$$

then eliminate $i$ from $I$; else if

$$
\sum_{j=1}^{r}\left(Y_{i \ell j}-q_{\ell}\right) \leq-R\left(r ; \epsilon_{\ell}, h_{1}^{2}, S_{i \ell}^{2}\right)
$$

then add $\ell$ to $S_{i}$.

For each $i \in I$, if $\left|S_{i}\right|=s$, then move $i$ from $I$ to $F$.

Stopping Rule: If $|I|=0$, then return $F$ as a set of feasible systems.

Otherwise, take one additional observation $\mathbf{Y}_{i, r+1}$ from each system $i \in I$ and let $Y_{i, r+1}^{a}=\mathbf{A}^{\prime} \mathbf{Y}_{i, r+1}$. Set $r=r+1$ and go to Feasibility Check.

Batur and $\operatorname{Kim}(2005)$ show that $\mathcal{F}_{\mathcal{A}}$ guarantees CD with probability at least $1-\left(\alpha_{0}+\alpha_{1}\right)$. When the overall nominal confidence level is $1-\alpha$, choosing $\alpha_{0}$ and $\alpha_{1}$ such that $\alpha_{0}+\alpha_{1}=\alpha$ guarantees $\mathrm{CD}$ with probability at least $1-\alpha$. However, with this choice of $\alpha_{0}$ and $\alpha_{1}, \mathcal{F}_{\mathcal{A}}$ might not perform better than $\mathcal{F}_{\mathcal{B}}$ under all circumstances. For example, suppose that the overall nominal confidence level is 95\% and we choose $\alpha_{0}=\alpha_{1}=0.025$. If all the systems are in the desirable region $D$, elimination based on aggregated observations is not likely to be utilized and $\mathcal{F}_{\mathcal{A}}$ becomes very similar to $\mathcal{F}_{\mathcal{B}}$ except that $\mathcal{F}_{\mathcal{A}}$ uses a larger confidence level $1-\alpha_{1}=0.975$ instead of $1-\alpha=0.95$. Thus, the performance of $\mathcal{F}_{\mathcal{A}}$ is likely to be worse than $\mathcal{F}_{\mathcal{B}}$ in this situation. However, it is possible that $\mathcal{F}_{\mathcal{A}}$ performs better than $\mathcal{F}_{\mathcal{B}}$ if there are a number of acceptable or unacceptable systems in terms of the original constraints: the saving from eliminating acceptable or unacceptable systems earlier by the screening part with aggregated observations might be large enough to compensate using a larger probability $1-\alpha_{1}$ than $1-\alpha$ for the screening part for the original individual constraints.

On the other hand, if we choose $\alpha_{1}=\alpha$ and $0<\alpha_{0}$, then $\mathcal{F}_{\mathcal{A}}$ is guaranteed to perform better than $\mathcal{F}_{\mathcal{B}}$ in terms of the number of replications required until we reach a decision. However, the efficiency is achieved at the cost of PCD: the actual PCD for $\mathcal{F}_{\mathcal{A}}$ is now only guaranteed to be $\geq 1-\left(\alpha_{0}+\alpha_{1}\right)$ which is smaller than $1-\alpha$. However, we know that $\mathcal{F}_{\mathcal{B}}$ and $\mathcal{F}_{\mathcal{A}}$ are already quite conservative and the actual PCD is usually considerably larger than the theoretical lower bound $1-\left(\alpha_{0}+\alpha_{1}\right)$. So, if one chooses $0<\alpha_{0}<\alpha$ and $\alpha_{1}=\alpha$, it will certainly help $\mathcal{F}_{\mathcal{A}}$ perform better than $\mathcal{F}_{\mathcal{B}}$ while the actual PCD is still $\geq 1-\alpha$ most of the time.

\section{EXPERIMENTAL RESULTS}

In this section, we present some experimental results that show the performance of the $\mathcal{F}_{\mathcal{B}}$ and $\mathcal{F}_{\mathcal{A}}$ procedures. We test the cases of $k=1$ and 9 systems. We assume that there are five stochastic constraints $(s=5)$. For replication $j$ of system $i, i=1,2, \ldots, k$, we assume that $\mathbf{Y}_{i j}$ is multivariate normally distributed with equal marginal variance $\sigma_{i \ell}^{2}=1$, for $\ell=1,2, \ldots, s$, and equal correlation, $\rho$, for each pair of systems. We test $\rho=\{-0.15,0.0,0.3\}$. We choose these correlation values because a multivariate normal distribution requires a positive definite variance-covariance matrix by definition, and these are three of the correlation values that guarantee a positive definite variance-covariance matrix. We set $\mathbf{E}=\left[\epsilon_{\ell}\right]_{\ell=1,2, \ldots, s}$ where $\epsilon_{\ell}=1 / \sqrt{n_{0}}$ and $n_{0}=10$. Also, without loss of generality, we can assume that $\mathbf{q}=$ $(0,0, \ldots, 0)^{\prime}$. The overall nominal confidence $1-\alpha$ is set to $1-\alpha=0.95$. In the $\mathcal{F}_{\mathcal{A}}$ procedure, we set $\alpha_{0}=\alpha_{1}=0.05$. Note that the $\mathcal{F}_{\mathcal{A}}$ procedure with this choice of $\alpha_{0}$ and $\alpha_{1}$ only guarantees the actual $\mathrm{PCD} \geq 0.90$.

The systems, used in the experiments, are categorized into $S_{D}, S_{A}, S_{U}$ depending on its mean configurations on $\mathbf{y}_{i}$. The mean configurations for a system in each region are given in Table 1. We have three desirable, three acceptable, and three unacceptable mean configurations. D1 is more difficult-requires more observations - than D2 and D2 is more difficult than D3. Similarly, A1 (or U1) is more difficult than A2 (or U2) and A2 (or U2) is more difficult than A3 (or U3). For example, a system with the D1 configuration has performance measures that are all exactly equal to $-\epsilon$ and barely falls into the desirable region $D$. On the other hand, all performance measures of a system with the D3 configuration are much smaller than $-\epsilon$ and the system is clearly a desirable system in $D$. 
Table 1: The Mean Configurations of the Performance Measures Associated with $s$ Stochastic Constraints

\begin{tabular}{|c|c|}
\hline & desirable \\
\hline D1 & $y_{\ell}=-\epsilon, \ell=1,2, \ldots, s$ \\
\hline D2 & $y_{\ell}=-\ell \epsilon, \ell=1,2, \ldots, s$ \\
\hline D3 & $y_{\ell}=-10 \epsilon, \ell=1,2, \ldots, s$ \\
\hline & acceptable \\
\hline A1 & $y_{1}=y_{2}=-2 \epsilon, y_{\ell}=-\epsilon / 2, \ell=3,4, \ldots, s$ \\
\hline A2 & $y_{\ell}=0, \ell=1,2, \ldots, s$ \\
\hline A3 & $y_{\ell}=\epsilon / 2, \ell=1,2, \ldots, s$ \\
\hline & unacceptable \\
\hline U1 & $y_{1}=y_{2}=-2 \epsilon, y_{\ell}=\epsilon, \ell=3,4, \ldots, s$ \\
\hline U2 & $y_{\ell}=\epsilon, \ell=1,2, \ldots, s$ \\
\hline U3 & $y_{\ell}=\ell \epsilon, \ell=1,2, \ldots, s$ \\
\hline
\end{tabular}

Table 2: Sample Average of Total Number of Replications Required in $\mathcal{F}_{\mathcal{B}}$ and $\mathcal{F}_{\mathcal{A}}$ when $k=1, s=5$, and $\sigma_{i \ell}^{2}=1$ for all $i$ and $\ell$

\begin{tabular}{|c|c|c|c|c|c|c|}
\hline & \multicolumn{2}{|c|}{$\rho=-0.15$} & \multicolumn{2}{c|}{$\rho=0.0$} & \multicolumn{2}{c|}{$\rho=0.3$} \\
\cline { 2 - 7 } & $\mathcal{F}_{\mathcal{B}}$ & $\mathcal{F}_{\mathcal{A}}$ & $\mathcal{F}_{\mathcal{B}}$ & $\mathcal{F}_{\mathcal{A}}$ & $\mathcal{F}_{\mathcal{B}}$ & $\mathcal{F}_{\mathcal{A}}$ \\
\hline D1 & 72 & 71 & 71 & 71 & 68 & 66 \\
\hline D2 & 47 & 47 & 47 & 47 & 46 & 46 \\
\hline D3 & 11 & 11 & 11 & 11 & 11 & 11 \\
\hline A1 & 87 & 87 & 85 & 85 & 82 & 81 \\
\hline A2 & 49 & 22 & 52 & 26 & 60 & 37 \\
\hline A3 & 27 & 10 & 28 & 11 & 31 & 17 \\
\hline U1 & 25 & 20 & 25 & 20 & 27 & 24 \\
\hline U2 & 19 & 10 & 20 & 10 & 22 & 12 \\
\hline U3 & 11 & 10 & 11 & 10 & 11 & 10 \\
\hline
\end{tabular}

In tables, we report the sample average of the total number of replications rather than the sample average of total number of observations corresponding to each constraint since a vector $\mathbf{Y}_{i j}$ can be obtained at the same time whenever a replication of system $i$ is finished.

Tables 2 and 3 show the sample average of total number of replications and the corresponding estimated PCD in $\mathcal{F}_{\mathcal{B}}$ and $\mathcal{F}_{\mathcal{A}}$ when $k=1, s=5$, and $\sigma_{i \ell}^{2}=1$. These estimated values are obtained as a result of 10,000 macroreplications (complete repetitions). For a system with the D1, D2, or D3 configuration, both procedures require about the same number of total number of replications as expected, because (i) the value of $\alpha$ of the $\mathcal{F}_{\mathcal{B}}$ is same as the value of $\alpha_{1}$ of $\mathcal{F}_{\mathcal{A}}$; and (ii) the screening with aggregated observations of the $\mathcal{F}_{\mathcal{A}}$ procedure is not likely to be utilized. For the A1, A2, A3, U1, U2, or U3 configuration, we observe a significant decrease in the total number of replications required in the $\mathcal{F}_{\mathcal{A}}$ procedure compared to the $\mathcal{F}_{\mathcal{B}}$ procedure. However, there is some degradation in PCD. For example, for the D1 configuration, the actual PCD is around 0.93.

When nine systems-one system from each mean configuration from D1 through U3 - are considered, we do not observe degradation in PCD any more. For example, when
Table 3: Estimated PCD in $\mathcal{F}_{\mathcal{B}}$ and $\mathcal{F}_{\mathcal{A}}$ when $k=1$, $s=5$, and $\sigma_{i \ell}^{2}=1$ for all $i$ and $\ell$

\begin{tabular}{|c|c|c|c|c|c|c|}
\hline \multirow{2}{*}{} & \multicolumn{2}{|c|}{$\rho=-0.15$} & \multicolumn{2}{c|}{$\rho=0.0$} & \multicolumn{2}{c|}{$\rho=0.3$} \\
\cline { 2 - 7 } & $\mathcal{F}_{\mathcal{B}}$ & $\mathcal{F}_{\mathcal{A}}$ & $\mathcal{F}_{\mathcal{B}}$ & $\mathcal{F}_{\mathcal{A}}$ & $\mathcal{F}_{\mathcal{B}}$ & $\mathcal{F}_{\mathcal{A}}$ \\
\hline D1 & 0.961 & 0.961 & 0.962 & 0.952 & 0.961 & 0.930 \\
\hline D2 & 0.992 & 0.992 & 0.993 & 0.993 & 0.992 & 0.992 \\
\hline D3 & 1.000 & 1.000 & 1.000 & 1.000 & 1.000 & 1.000 \\
\hline U1 & 1.000 & 1.000 & 1.000 & 1.000 & 1.000 & 1.000 \\
\hline U2 & 1.000 & 1.000 & 1.000 & 1.000 & 1.000 & 1.000 \\
\hline U3 & 1.000 & 1.000 & 1.000 & 1.000 & 1.000 & 1.000 \\
\hline
\end{tabular}

$\rho$ equals 0.0 , the $\mathcal{F}_{\mathcal{B}}$ procedure spends 764 total replications on average and provides the estimated PCD of 0.993 ; however, the $\mathcal{F}_{\mathcal{A}}$ procedure spends only 589 replications on average with the estimated PCD equal to 0.991. But when all the systems are in the desirable region $D$, both procedures show similar performance as expected although we did not include the results in this paper. In practice when $k$ is large, it is very rare that all the systems are in the desirable region $D$. Therefore, the $\mathcal{F}_{\mathcal{A}}$ procedure is expected to be more efficient than the $\mathcal{F}_{\mathcal{B}}$ procedure in general with very little or no degradation in PCD.

\section{CONCLUSION}

In a discrete optimization problem with several stochastic constraints, the first step is to identify the set of feasible or near-feasible systems and the proposed procedures in the paper will be useful in that step. In this paper, we show that a feasibility detection procedure due to Andradóttir, Goldsman, and Kim (2005) for a single stochastic constraint can easily be extended to multiple constraints. Also, we show that the extended procedure can be further improved by combining it with a screening procedure based on aggregated observations. Finally, Batur and Kim (2005) provide lemmas that generalize the extension from a single constraint to multiple constraints and that constructs combined procedures with the acceleration technique for any feasibility check procedure that satisfies a certain set of conditions.

\section{ACKNOWLEDGMENT}

This material was supported by the National Science Foundation under Grant Number DMI-0400260.

\section{REFERENCES}

Andradóttir, S., D. Goldsman, and S.-H. Kim. 2005. Fully sequential procedures for comparing constrained systems via simulation. To appear in Proceedings of the 2005 Winter Simulation Conference, ed. M. E. Kuhl, N. M. Steiger, F. B. Armstrong, and J. A. Joines. Institute of Electrical and Electronics Engineers, Piscataway, New Jersey. 
Batur, D. and S.-H. Kim. 2005. Finding a set of feasible systems when the number of systems or constraints is large. Working paper, School of Industrial and Systems Engineering, Georgia Institute of Technology, Atlanta, Georgia.

Butler, J., D. J. Morrice, and P. W. Mullarkey. 2001. A multiple attribute utility theory approach to ranking and selection. Management Science, 47:800-816.

Santner, T. J., and A. C. Tamhane. 1984. Designing experiments for selecting a normal population with a large mean and a small variance. In Design of Experiments - Ranking and Selection: Essays in Honor of Robert E. Bechhofer, ed. T. J. Santner and A. C. Tamhane, 179-198. New York: Marcel-Dekker.

\section{AUTHOR BIOGRAPHIES}

DEMET BATUR is a Ph.D. candidate in the School of Industrial and Systems Engineering at the Georgia Institute of Technology. She received her B.Sc. degree in Industrial Engineering from Marmara University, Istanbul, Turkey, and her M.S. degree in School of Industrial and Systems Engineering from Georgia Institute of Technology, Atlanta, GA in 2001 and 2004, respectively. Her current research interests include ranking and selection and simulation output analysis. Her e-mail address is <dbatur@isye.gatech.edu>.

SEONG-HEE KIM is an Assistant Professor in the School of Industrial and Systems Engineering at the Georgia Institute of Technology. She received her Ph.D. in Industrial Engineering and Management Sciences from Northwestern University in 2001. Her research interests include ranking and selection, quality control, and simulation output analysis. She is a member of INFORMS and IIE, and serves the editorial board of the Simulation Department of IIE Transactions. Her e-mail and web addresses are <skim@isye. gatech. edu $>$ and $<w w w$. isye.gatech.edu/ skim/>, respectively. 